Otaliba Libânio de Morais Neto 1 Marilisa Berti de Azevedo Barros ${ }^{2}$ Celina Maria Turchi Martelli 1 Simonne Almeida e Silva 1 Suzana Marta Cavenaghi 3 João Bosco Siqueira Jr. 4

\section{Diferenças no padrão de ocorrência da mortalidade neonatal e pós-neonatal no Município de Goiânia, Brasil, 1992-1996: análise espacial para identificação das áreas de risco}

\author{
Differential patterns of neonatal and post-neonatal \\ mortality rates in Goiânia, Brazil, 1992-1996: \\ use of spatial analysis to identify high-risk areas
}

1 Instituto de Patologia Tropical e Saúde Pública, Universidade Federal de Goiás. Rua Delenda Rezende de Melo s/n, Goiânia, GO 74605-050,Brasil.

2 Departamento de Medicina Preventiva e Social, Faculdade de Ciências Médicas, Universidade Estadual de Campinas. C. P. 6111, Campinas, SP 13083-970, Brasil.

3 Núcleo de Estudos Populacionais, Universidade Estadual de Campinas.

C. P. 6166, Campinas, $S P$ 13081-970, Brasil.

4 Secretaria Municipal de Saúde de Goiânia. Av. 5a Radial, 9 216-A Lote 5 , Setor Pedro Ludovico, Goiânia, GO

74 823-030, Brasil.

\begin{abstract}
The aim of this study was to investigate the spatial pattern of neonatal and postneonatal mortality in the city of Goiânia, Central Brazil. Analyses were based on linked birth and death certificates relating to 101,000 in-hospital live births from mothers residing in the city of Goiânia over the 1992-1996 period. Overall neonatal and post-neonatal mortality probabilities were calculated using the linked database. The empirical Bayes method was applied to smooth the estimated rates and minimize random fluctuation. Spatial units of analysis were 65 urban districts, corresponding to the urban planning sectors. The following exploratory spatial analyses were applied: "global" Moran's I statistic, local Moran LISA map, and Gi* local statistics. For both neonatal and post-neonatal mortality there was statistically significant spatial autocorrelation. Results of post-neonatal mortality showed a high-risk cluster located on the outskirts of the city. For the neonatal period, a heterogeneous mortality pattern was found with high-risk districts in all regions, including central areas.
\end{abstract}

Key words Spatial Analysis; Infant Mortality; Neonatal Mortality

Resumo Este artigo refere-se à pesquisa acerca do padrão espacial dos componentes neonatal e pós-neonatal da mortalidade infantil em Goiânia, no Estado de Goiás, Brasil. A população do estudo foi a coorte de 101 mil nascidos vivos, residentes em Goiânia, de 1992 a 1996. As probabilidades de morte infantil foram estimadas mediante o cotejo dos arquivos de óbitos e de nascidos vivos. Para minimizar as flutuações aleatórias das taxas, empregou-se o método Bayesiano empírico. A unidade de análise do padrão espacial foi constituída pelos 65 distritos urbanos de planejamento. Para análise de autocorrelação espacial foram utilizados: Moran "global", Moran local e estatística $\mathrm{Gi}^{*}$ local. Os componentes neonatal e pós-neonatal da mortalidade infantil evidenciaram autocorrelação espacial estatisticamente significativa. No período pós-neonatal, os distritos de risco concentram-se nas regiões periféricas do município. No período neonatal, o padrão de ocorrência é heterogêneo, havendo distritos de alto risco distribuídos em todas as regiões, inclusive na região Central de Goiânia.

Palavras-chave Análise Espacial; Mortalidade Infantil; Mortalidade Neonatal 


\section{Introdução}

O estudo da distribuição da mortalidade infantil no espaço geográfico de áreas com elevado grau de urbanização é instrumento útil para o desencadeamento de intervenções de saúde destinadas à população materno-infantil. Dentre as contribuições desta abordagem, destacam-se: (a) monitoramento de eventos e riscos de morte em pequenas áreas - bairros, distritos de saúde etc. -, apresentando os resultados na forma de Figuras de fácil leitura e interpretação; (b) identificação de grupos de população expostos a altos riscos de mortalidade infantil e (c) identificação e compreensão dos fatores associados aos riscos de mortalidade infantil e seus componentes (Kelly, 1999; Lawson et al., 1999).

O espaço urbano é categoria analítica que expressa uma síntese entre os momentos da produção e os da reprodução social. Nestas circunstâncias, as relações sociais, os processos de urbanização, a industrialização, a migração e os padrões de consumo tanto individual quanto coletivo de uma população configuram a ocupação do espaço urbano com certo grau de homogeneidade social em determinado contexto histórico (Duarte, 1992; Paim, 1997; Santos, 1996).

A maioria dos estudos brasileiros analisa os padrões de distribuição da mortalidade infantil segundo duas preocupações centrais: (a) identificar áreas constituídas de grupos populacionais sob alto risco de morte infantil e (b) correlacionar os riscos de mortalidade infantil com variáveis socioeconômicas e de assistência à saúde, medidas enquanto variáveis de um agregado (Guimarães \& Fischmann, 1986; Monteiro, 1982; Monteiro et al., 1980; Paim \& Costa, 1987, 1993; Souza et al., 1999). Nesses trabalhos, é rara a utilização de técnicas de análise espacial propriamente dita para representação e análise dos mapas de risco de mortalidade infantil e de seus componentes neonatal e pósneonatal.

O mapeamento de doenças vem sendo instrumento básico no campo da saúde pública e, em anos recentes, muitos avanços nas técnicas de análise têm sido desenvolvidos com o objetivo de produzir mapas cuja construção deve estar livre de "ruídos aleatórios" ou de artefatos relacionados à extensão da área geográfica e à população existente nas regiões enfocadas (Bailey \& Gatrell, 1995; Lawson et al., 1999).

Dentre as técnicas de análise de dados espaciais que podem ser empregadas no estudo do padrão de distribuição espacial da mortalidade infantil, é possível ressaltar a análise ex- ploratória de dados espaciais, que visa descrever e explicar como o padrão de distribuição da mortalidade infantil se expressa no espaço geográfico, ou seja, procura verificar se existe dependência espacial na determinação do padrão da mortalidade infantil e quais as relações espaciais presentes nesta determinação (Bailey \& Gatrell, 1995).

Em publicação anterior (Morais Neto \& Barros, 2000) foram investigados os fatores de risco individuais para os componentes neonatal e pós-neonatal da mortalidade infantil na coorte de nascidos vivos no Município de Goiânia, Estado de Goiás, Brasil. No presente artigo, os objetivos são investigar o padrão espacial da mortalidade neonatal e pós-neonatal e produzir mapas que identifiquem áreas de risco para os dois componentes da mortalidade infantil no espaço urbano de Goiânia mediante técnicas de análise de dados espaciais.

\section{Material e métodos}

\section{Área e população de estudo}

Este estudo foi conduzido em Goiânia, Estado de Goiás, Brasil. O Município de Goiânia está localizado nas coordenadas geográficas 490 00' - 49o 45’W e 16o 30' - 17o 00’S, apresenta área de $789,87 \mathrm{~km}^{2}$ e conta com população de um milhão de habitantes, cuja densidade populacional é de 1.252,7 hab. $/ \mathrm{km}^{2}$.

A população do estudo foi compreendida pela coorte de 101 mil nascidos vivos que tiveram declaração de nascido vivo preenchida entre os anos de 1992 e 1996.

\section{Fontes de dados}

A fonte de dados a respeito dos nascidos vivos é a declaração de nascido vivo (DNV) - documento base do sistema de informações sobre nascidos vivos (SINASC) -, implantada no País em 1990 (Mello-Jorge et al., 1993). As informações foram coletadas no Departamento de Morbimortalidade da Secretaria Estadual de Saúde e no Núcleo de Informações em Saúde da Secretaria Municipal de Saúde de Goiânia. A coorte retrospectiva de nascidos vivos é formada pelos nascidos em Goiânia no período de 1992 a 1996, cujas mães residiam no município. O total de nascidos vivos da coorte é de aproximadamente 101 mil, o que representa cobertura de 90\% segundo o número esperado de nascidos vivos para o município (Mello-Jorge et al., 1996).

Os óbitos infantis foram estabelecidos pelo número total de óbitos no período neonatal 
(antes de 28 dias de vida) e no período pós-neonatal (entre 28 e 365 dias de vida). A fonte de dados utilizada para a obtenção dos óbitos infantis foi a declaração de óbito (DO) documento base do sistema de informações de mortalidade (SIM) referentes aos anos de 1992 a 1997.

\section{Relacionamento dos bancos de dados}

Cada declaração de óbito foi pareada com a respectiva declaração de nascido vivo através dos seguintes procedimentos: (a) busca automática no arquivo de nascidos vivos, adotando-se campos chaves comuns à DNV e à DO - a saber: data de nascimento, sexo e bairro de residência -, identificando os prováveis códigos de DNV para cada óbito; (b) busca manual pelo nome da mãe para confirmar a etapa anterior; (c) busca nos cartórios de registro civil para os casos de insucesso nas etapas anteriores.

O sucesso do emparelhamento foi, em média, de $95 \%$ para as cinco coortes de nascimento. A partir desses procedimentos tornou-se possível a distinção, na coorte retrospectiva dos nascidos vivos, entre os que evoluíram para o óbito e os sobreviventes no primeiro ano de vida, bem como o cálculo das probabilidades de morte no primeiro ano de vida e nos períodos neonatal e pós-neonatal, que constituíram as taxas a serem empregadas na análise espacial de dados.

\section{Banco de dados tabulares}

A partir do banco de dados oriundo do procedimento de confronto entre os arquivos de óbitos e de nascidos vivos foi construído um banco de dados em que a variável indicadora consistiu no código do distrito urbano de planejamento, enquanto as demais variáveis foram: nascidos vivos totais, óbitos infantis no período neonatal, óbitos infantis no período pósneonatal, probabilidade de morte no período neonatal e probabilidade de morte no período pós-neonatal. Na construção dos bancos de dados recorreu-se ao programa Epi Info 6.04 (CDC/WHO, 1996).

\section{Geo-referenciamento}

Os eventos - óbitos infantis e nascidos vivos sobreviventes - foram geocodificados nos distritos urbanos de planejamento. A área urbana do Município de Goiânia é dividida em 65 distritos urbanos de planejamento, definidos com base em critérios de homogeneidade socioeconômica, planejamento urbano e características físico-territoriais (IPLAN, 1992). Foram excluí- dos do processo de análise: os distritos 44 e 46, correspondentes ao aeroporto e à Central de Abastecimento de Goiânia (CEASA), respectivamente; os distritos 22 e 63, em razão do pequeno número de nascidos vivos (menos de 100 nos cinco anos de estudo); o distrito 62, por ter-se constituído em município independente; e, por fim, o distrito número 65, em virtude de caracterizar-se por adensamento populacional recente e ter sido acrescido à divisão territorial apenas em 1996.

\section{Mapas digitais}

Fez-se uso do mapa urbano básico digital de Goiânia, que foi produzido pela Companhia de Processamento de dados de Goiânia (COMDATA) e adaptado para uso em Sistemas de Informações Geográficas e análise de dados espaciais em projetos em andamento no Departamento de Medicina Tropical, Saúde Coletiva e Dermatologia da Universidade Federal de Goiânia (UFG) (Ximenes et al., 1999).

\section{Delineamento do estudo}

Para cumprir os objetivos do artigo, delineouse um estudo ecológico do tipo exploratório por intermédio da aplicação de técnicas de análise exploratória de dados espaciais. Foram utilizados o mapa digital básico de Goiânia e os sistemas de informações geográficas ArcView (ESRI, 1996), além dos aplicativos de análise espacial Info-Map (Bailey \& Gatrell, 1995) e Spacestat (Anselin, 1995).

\section{- Análise de dados}

Empregou-se a estratégia de análise de dados de área. Foram utilizadas técnicas de análise espacial de dados voltadas para: (a) sumarização dos dados: mapas das probabilidades de morte observadas nos períodos neonatal e pósneonatal e das taxas Bayesianas. A aplicação das taxas Bayesianas teve como objetivo minimizar a instabilidade das probabilidades de morte, eliminando a flutuação aleatória presente em pequenas áreas (Assunção et al., 1998; Bailey \& Gatrell, 1995; Marshal, 1991). O método usado para a construção das taxas Bayesianas foi o método Bayesiano empírico proposto por Marshal (1991), utilizando o método de momentos, em que a taxa de cada distrito urbano é ajustada levando-se em conta as taxas de seus vizinhos; (b) análise exploratória de dados espaciais: (1) investigação da autocorrelação espacial global, lançando-se mão do índice I de Moran sob as suposições de normalidade e de 
randomização; (2) análise dos indicadores de autocorrelação local - Moran local e estatística Gi* (Anselin, 1999), utilizando-se as estimativas Bayesianas das probabilidades de morte nos períodos neonatal e pós-neonatal. Para a análise de autocorrelação adotou-se, como critério para construção da matriz de vizinhança, o compartilhamento de bordas entre os distritos (vizinhos de primeira ordem).

\section{Resultados}

As probabilidades médias de morte observadas para os períodos neonatal e pós-neonatal são respectivamente 10,9 (variância $=32,8$ ) e 5,3 (variância $=9,3$ ). Na estimativa Bayesiana empírica das probabilidades vê-se que as médias são semelhantes $(11,4$ no período neonatal e 5,5 no pós-neonatal), mas há diminuição da variância das medidas (11,9 no período neonatal e 3,3 no pós-neonatal) em virtude da redução das flutuações aleatórias dos riscos de mortalidade nos distritos.

A visualização dos riscos de morte neonatal na Figura 1a mostra que os distritos que compõem o quartil de maior risco abrangem praticamente todas as regiões do município. Destaca-se a presença do distrito 56 (região Leste) como outlier de alto risco. A Figura $1 b$ apresenta as estimativas Bayesianas da probabilidade de morte no período neonatal. Nesta figura nota-se que os maiores riscos concentram-se nos distritos 30, 31, 33, 35, 36, 14 e 8 (regiões Noroeste e Mendanha), no distrito 56 (região Leste), nos distritos 7, 49 e 54 (região Central), no distrito 42 (região Norte) e no distrito 12 (região Sul), ao passo que os menores riscos, nos distritos 1, 2, 3, 4 e 11 da região Central e em alguns distritos específicos das demais regiões de Goiânia.

No período pós-neonatal, o mapa da mortalidade observada (Figura 2a) expõe que os maiores riscos de morte estão concentrados nos distritos periféricos nas regiões Noroeste, Mendanha, Oeste, Norte e Leste. O mapa com estimativas Bayesianas assinala concentração ainda maior dos riscos nos distritos que constituem as regiões periféricas (Noroeste, Mendanha, Oeste, Norte e Leste) e, ao mesmo tempo, a concentração dos distritos de baixo risco na região Central da cidade (Figura $2 b$ ).

Os resultados da autocorrelação espacial global (estatística I de Moran) - calculados segundo a aproximação normal e assumindo-se uma distribuição aleatória (suposição de randomização) - mostram, para o período pósneonatal, autocorrelação espacial global estatisticamente significativa para ambas as taxas - probabilidade de morte observada e estimativa Bayesiana empírica. No período neonatal, não se observa autocorrelação estatisticamente significativa na escala dos distritos urbanos de planejamento, considerando-se a matriz de vizinhos de primeira ordem.

Com o objetivo de avaliar se o distrito 56 outlier de alto risco circundado de vizinhos de riscos relativamente baixos - influencia o cálculo do coeficiente de autocorrelação global de forma a torná-lo não significativo, foi refeita a análise de autocorrelação global sem a interferência do outlier. Para minimizar a interferência do outlier, substituiu-se a probabilidade de morte do distrito 56 por taxa média de seus vizinhos. A Tabela 1 mostra os resultados oriundos da nova análise. A probabilidade de morte observada manteve autocorrelação espacial não significativa no período neonatal, enquanto a estimativa bayesiana apresentou a estatística I de Moran estatisticamente significativa ( $\mathrm{I}=0,17, \mathrm{p}=0,03$ ) para ambos os métodos de cálculo - suposições de normalidade e de randomização -, mostrando a existência de padrão de dependência espacial para a mortalidade infantil no período neonatal na escala dos distritos urbanos. Assumindo este padrão como o de maior poder explicativo para os dados, partiu-se para a análise de autocorrelação local.

O exame da autocorrelação local mediante o Moran local (Figura 3a) mostra que existem, para o período neonatal, três áreas com índices estatisticamente significativos: duas de alto risco - distritos 31, 32, 33, 35 e 36 (região Mendanha/Noroeste) e distrito 54 (região Central) - e uma, de baixo risco - distrito 3 (região Central). A estatística Gi* identifica o cluster de alto risco da região Mendanha/Noroeste assim como o de baixo risco da região Central (Figura 4a).

Para o período pós-neonatal, os exames da autocorrelação local - tanto o Moran local quanto a estatística $\mathrm{Gi}^{*}$ - identificam dois grandes clusters de alto risco; o primeiro, formado pelos distritos das regiões Mendanha e Noroeste e o segundo, pelos da região Oeste, da mesma forma que um cluster de baixo risco constituído pela quase totalidade dos distritos da região Central da cidade (Figuras 3 b e 4 b).

\section{Discussão}

Existem poucos estudos realizados no Brasil que investigaram o padrão espacial da mortalidade infantil e seus componentes, bem como o da ocorrência de baixo peso ao nascer, por meio do emprego de técnicas de análise espacial (Campos, 1997; d'Orsi \& Carvalho, 1998; Leal \& Szwarcwald, 1997). O estudo de Campos 

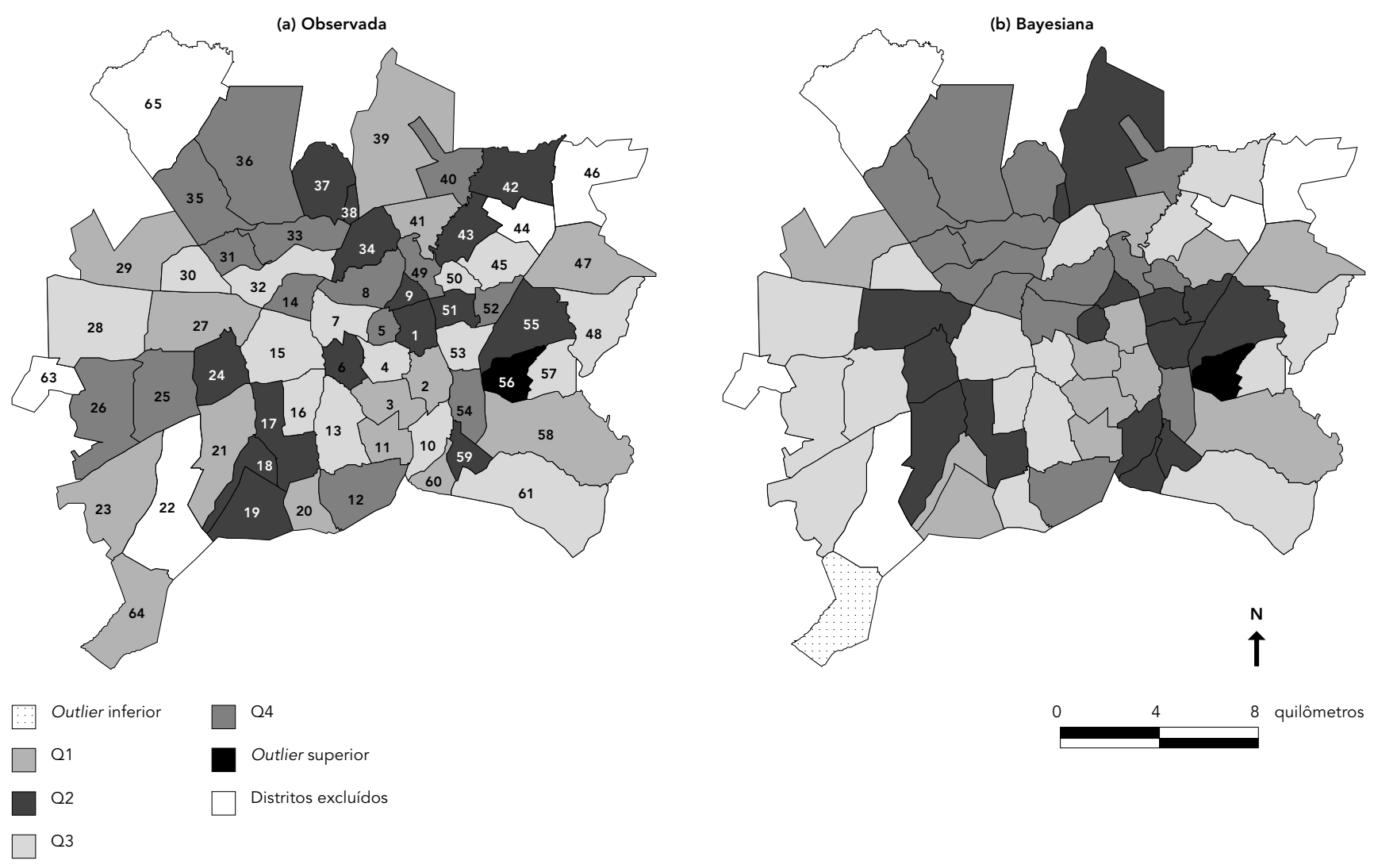

(1997), que utilizou bairros de residência, como unidade de análise observou distribuição espacial aleatória da mortalidade neonatal no Município do Rio de Janeiro. O estudo de Leal \& Szwarcwald (1997) registrou a existência de autocorrelação espacial durante o período neonatal tardio (7-27 dias de vida) nos municípios do Estado do Rio de Janeiro de 1979 a 1981 e a distribuição espacial aleatória, de 1990 a 1992. Segundo as autoras, houve homogeneização dos riscos de morte neonatal tardia nos municípios do Rio de Janeiro entre os dois períodos analisados no contexto de queda da mortalidade neonatal. D’Orsi \& Carvalho (1998), investigando o padrão espacial do baixo peso ao nascer nos bairros do Município do Rio de Janeiro, observaram distribuição aleatória do baixo peso ao nascer, considerado um dos principais mediadores na ocorrência dos óbitos neonatais.
Os resultados apresentados no presente artigo mostram a importância da utilização de dados de nascidos vivos de cinco coortes de nascimentos (1992-1996) e da construção de estimativas Bayesianas das probabilidades de morte neonatal e pós-neonatal para reduzir a instabilidade das estimativas das probabilidades de morte observadas, minimizando as flutuações aleatórias existentes nos riscos de morte nos distritos urbanos que apresentam pequeno número de nascidos vivos. Como pode ser visto nos resultados, a suavização das probabilidades de morte pelo método Bayesiano empírico proporcionou a redução da variabilidade das taxas e melhor visualização da distribuição espacial dos riscos de morte.

Uma desvantagem da utilização de estimativa Bayesiana empírica local das probabilidades de morte infantil é o potencial de induzir a autocorrelação espacial. Esse efeito 

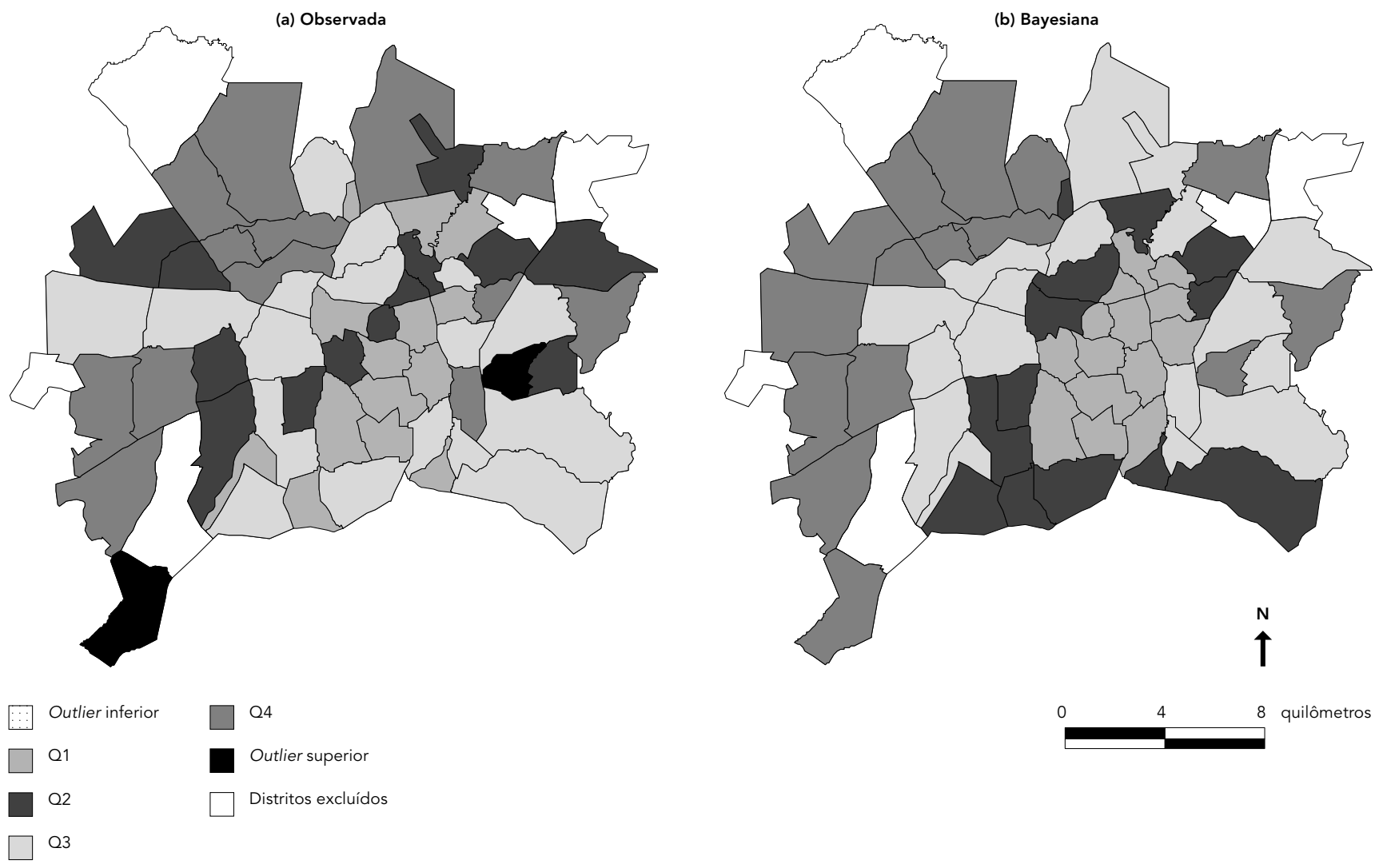

Tabela 1

Análise de autocorrelação espacial global da probabilidade de morte observada e estimativa Bayesiana empírica nos períodos neonatal e pós-neonatal. Distritos urbanos de Goiânia, 1992-1996.

\begin{tabular}{|c|c|c|c|c|}
\hline \multirow[t]{2}{*}{ Período/Variável } & \multicolumn{2}{|c|}{$\begin{array}{l}\text { Moran (suposição } \\
\text { normalidade) }\end{array}$} & \multicolumn{2}{|c|}{$\begin{array}{l}\text { Moran (suposição } \\
\text { randomização) }\end{array}$} \\
\hline & I & $\mathrm{p}$ & 1 & $\mathrm{p}$ \\
\hline \multicolumn{5}{|l|}{ Período Neonatal } \\
\hline Prob. Morte observada & 0,0230 & 0,63 & 0,0230 & 0,63 \\
\hline Estimativa Bayesiana & 0,1699 & 0,03 & 0,1699 & 0,03 \\
\hline \multicolumn{5}{|l|}{ Período Pós-Neonatal } \\
\hline Prob. Morte observada & 0,2324 & 0,003 & 0,2324 & 0,003 \\
\hline Estimativa Bayesiana & 0,7052 & $<0,001$ & 0,7052 & $<0,001$ \\
\hline
\end{tabular}

pode ocasionar superestimativa dos coeficientes de autocorrelação global e local nas áreas com pequenos números de nascidos vivos, onde o alisamento das taxas do distrito em direção à média de seus vizinhos é mais acentuada. Neste artigo, embora reconhecendo esse viés potencial, optou-se pelo recurso às estimativas Bayesianas para correção das taxas de mortalidade infantil em razão da coerência dos resultados obtidos com o perfil epidemiológico e socioeconômico dos distritos urbanos de Goiânia.

Na escala dos distritos urbanos de planejamento, a visualização das estimativas Bayesianas dos riscos da mortalidade neonatal e pósneonatal mostrou padrões distintos de distribuição espacial da mortalidade infantil segundo os seus componentes: (a) no período pósneonatal existe polaridade caracterizada por altos riscos nos distritos periféricos e baixos 


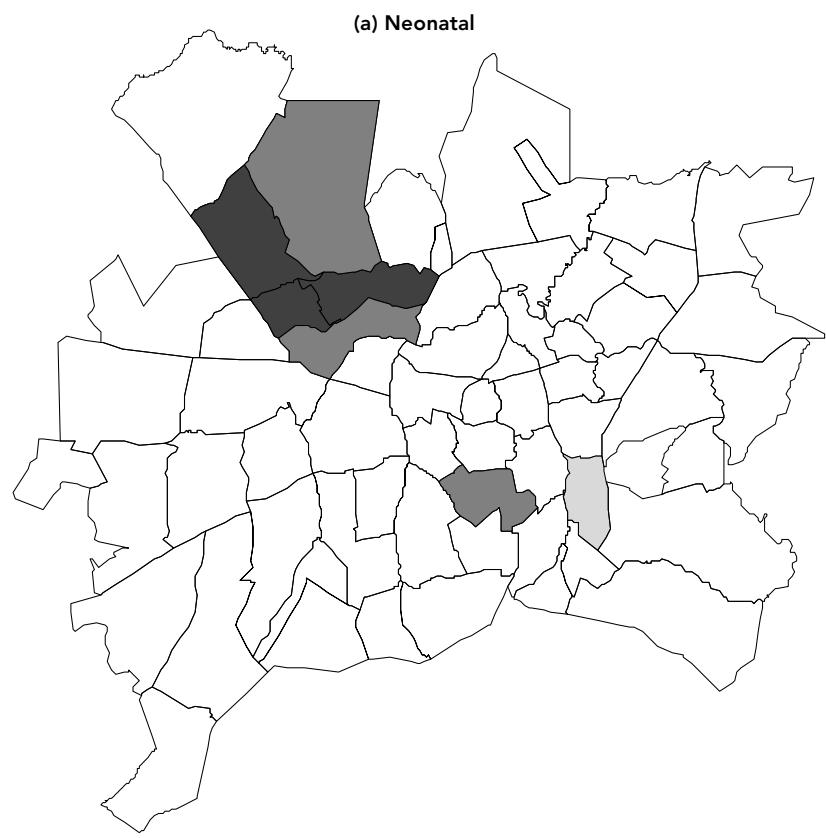

não significante

$p=0,05$

$p=0,01$

$p=0,001$

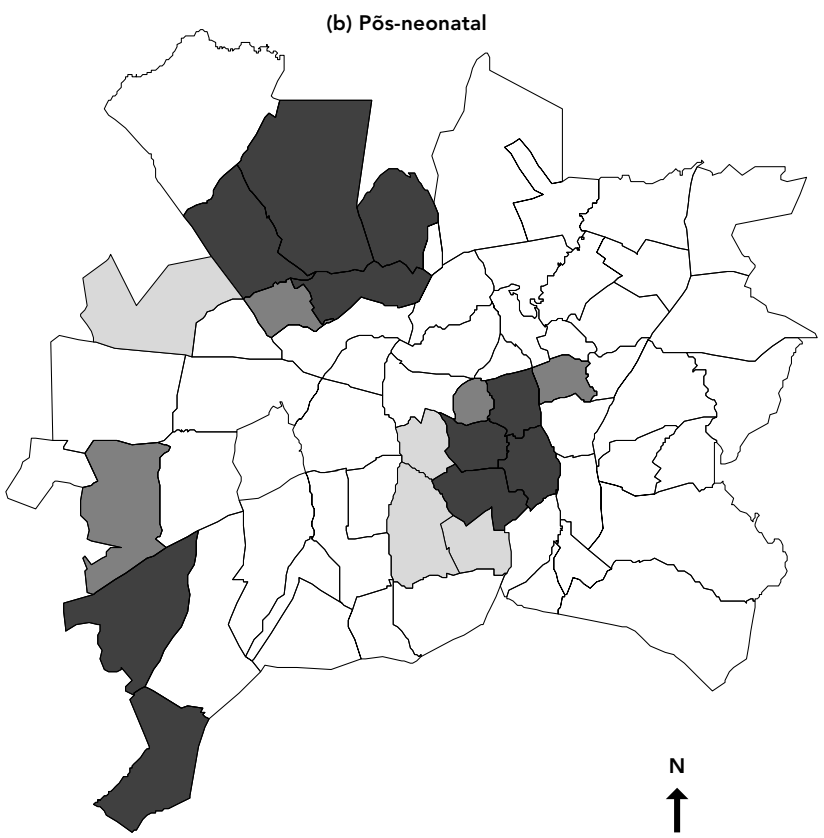

$\begin{array}{llll}0 & 4 & 8 & \text { quilômetros }\end{array}$ riscos nos distritos que formam a região Central (b) no período neonatal, o padrão espacial distinguiu-se pela ocorrência dos maiores riscos nos distritos periféricos que caracterizam a mortalidade no período pós-neonatal - em especial, nos distritos das regiões Mendanha/Noroeste -, mas também em distritos das regiões Central (distrito 54), Sul (distrito 12), Norte (distrito 42) e Meia Ponte (distrito 37).

O componente neonatal não se apresentou como evento espacialmente dependente ao utilizar-se o Moran global. No entanto, quando se retirou a influência do outlier de alto risco (distrito 56), a estimativa Bayesiana empírica da probabilidade de morte no período neonatal expressou autocorrelação estatisticamente significativa. Além disso, a análise dos indicadores de autocorrelação local mostrou a existência de duas áreas de alto risco: uma, na região Noroeste/Mendanha e outra, no distrito
54 da região Central, afora uma área de baixo risco na região Central da cidade.

Conforme Zoellner \& Schmidtmann (1999) e Tango (1999), a principal limitação do uso da estatística I de Moran é a sua utilização para doenças raras e para regiões com alta heterogeneidade da densidade populacional. No presente estudo, esta limitação foi reduzida com a utilização de uma coorte de nascidos vivos que abrangia um período de cinco anos.

Correlacionando-se o padrão espacial da mortalidade infantil com o processo de organização do espaço urbano do Município de Goiânia, pode-se afirmar que os distritos que constituem a área de maior risco de mortalidade no período pós-neonatal são os distritos de urbanização mais recente, surgidos no período da expansão urbana do município, ocorrida a partir do final da década de 70 e caracterizada pela proliferação de loteamentos irregulares, des- 


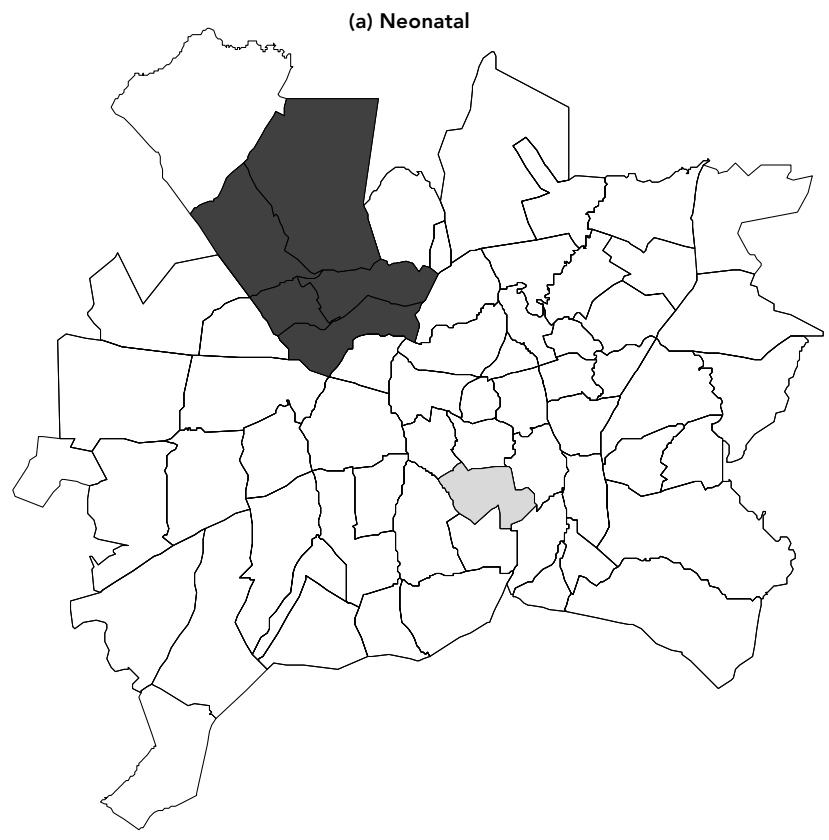

Baixo risco $(p<0,05)$

Não significante

Alto risco $(p<0,05)$

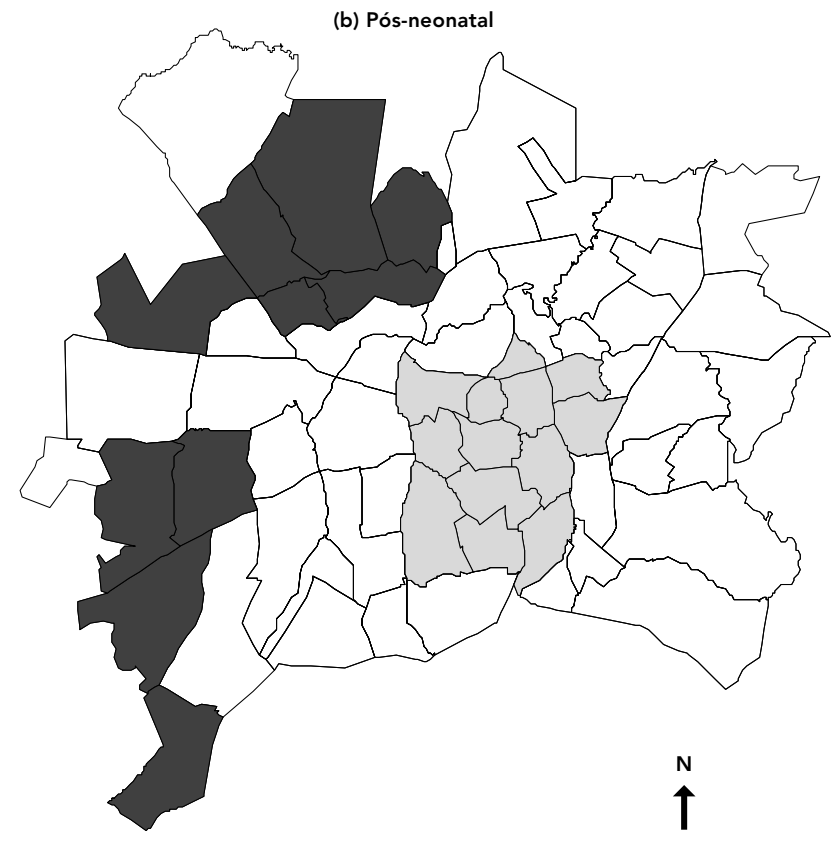

$0 \quad 4 \quad 8$ quilômetros providos de infra-estrutura básica e destinados à população de baixa renda, oriunda, em grande parte, da mobilidade intra-urbana e da migração (IPLAN, 1992). Com relação às áreas de maior risco de mortalidade no período neonatal, há sobreposição com os distritos de maior risco de mortalidade pós-neonatal da região Mendanha/Noroeste, afora outros, localizados nas regiões Central, Sul e Norte da cidade, que podem ser caracterizados como a "antiga" periferia da cidade. Estes distritos incluem alguns constituídos de população de baixa renda que vive em áreas de fundos de vale e outros, cuja população é heterogênea, do ponto de vista socioeconômico, em decorrência da coexistência de áreas faveladas e não faveladas em mesmo distrito. A população que reside nos distritos da "antiga" periferia, no entanto, diferencia-se da população dos distritos da área de expansão no que se refere ao maior acesso à infra-estru- tura urbana, às melhores condições de habitação, ao transporte urbano e, provavelmente, ao maior acesso à atenção básica de saúde. Os elevados riscos de mortalidade neonatal nos distritos da "antiga" periferia estão provavelmente relacionados a deficiências na qualidade da atenção pré-natal, ao parto e durante o período neonatal, tal como às dificuldades de acesso a tecnologias de maior custo cobertas parcialmente pelo Sistema Único de Saúde.

As diferenças observadas no padrão de ocorrência dos componentes neonatal e pós-neonatal da mortalidade infantil estão associadas à maior complexidade dos determinantes da mortalidade infantil no período neonatal. Os determinantes da mortalidade neonatal são resultantes da sobreposição entre os fatores socioeconômicos (saneamento, renda etc.) e os fatores relativos ao acesso e à qualidade da assistência a saúde no período neonatal, tais co- 
mo: assistência pré-natal, ao parto e ao recémnascido (Leal \& Szwarcwald, 1997).

A construção de mapas com menor instabilidade das medidas de riscos e a utilização de técnicas de estatística espacial para exame da autocorrelação espacial permitiram identificar os padrões de ocorrência da mortalidade neonatal e pós-neonatal no espaço urbano de Goiânia. A análise e interpretação destes Figuras

\section{Agradecimentos}

Agradecemos o apoio financeiro da Secretaria Municipal de Saúde de Goiânia e da Fundação de Apoio à Pesquisa da Universidade Federal de Goiás (FUNAPE).

\section{Referências}

ANSELIN, L., 1995. Spacestat. A Software Program for the Analysis of Spatial Data. Version 1.80. Morgantown: Regional Research Institute, West Virginia University.

ANSELIN, L., 1999. Spatial Data Analysis with Spacestat and ArcView. Workbook. 3rd Ed. <http://www. spacestat.com/>.

ASSUNÇÃO, R. M.; BARRETO, S. M.; GUERRA, H. L. \& SAKURAI, E., 1998. Figuras de taxas epidemiológicas: Uma abordagem Bayesiana. Cadernos de Saúde Pública, 14:713-723.

BAILEY, T. C. \& GATRELL, A. C., 1995. Interactive Spatial Data Analysis. Essex: Longman.

CAMPOS, T. P., 1997. Perfil de Nascimentos e Óbitos Infantis: A Busca da Assistência no Município do Rio de Janeiro. Dissertação de Mestrado, Rio de Janeiro: Escola Nacional de Saúde Pública, Fundação Oswaldo Cruz.

CDC (Centers for Disease Control and Prevention)/ WHO (World Health Organization), 1996. Epi Info 6, Versão 6.04: A Word Processor, Database and Statistics Program for Public Health. Atlanta: CDC/ Geneva: WHO.

D'ORSI, E. \& CARVALHO, M. S., 1998. Perfil de nascimentos no Município do Rio de Janeiro: Uma análise espacial. Cadernos de Saúde Pública, 14:367379.

DUARTE, C. M. R., 1992. Qualidade de vida e indicadores de Saúde: Aspectos da mortalidade infantil no Estado do Rio de Janeiro e suas regiões. Cadernos de Saúde Pública, 8:414-427. possibilitam: (a) identificar bolsões de nascidos vivos sob maior risco de morte neonatal e pós-neonatal; (b) subsidiar o planejamento e a programação de intervenções de saúde direcionadas à clientela materno-infantil e voltadas para a redução das desigualdades nos riscos de morte infantil, e (c) monitorar os riscos após as intervenções, possibilitando avaliação do impacto destas ações.
ESRI (Environmental Systems Research Institute Incorporation), 1996. ArcView GIS. The Geographic Information System for Everyone. Version 3.0. Redlands: ESRI.

GUIMARÃES, J. J. L. \& FISCHMANN, A., 1986. Desigualdades na mortalidade infantil entre favelados e não favelados no Município de Porto Alegre, Rio Grande do Sul, em 1980. Boletín de la Oficina Sanitaria Panamericana, 101:19-38.

IPLAN (Instituto de Planejamento Municipal), 1992. Plano de Desenvolvimento Integrado de Goiânia PDIG. Goiânia: IPLAN, Prefeitura de Goiânia.

KELLY, A., 1999. Case studies in Bayesian disease mapping for health and health service research in Ireland. In: Disease Mapping and Risk Assessment for Public Health (A. Lawson, A. Biggeri, D. Böhning, E. Lesaffre, J. Viel \& R. Bertollini, ed.) pp. 349-363, New York: John Wiley \& Sons.

LAWSON, A.; BÖHNING, D.; BIGGERI, A.; LESAFFRE, E. \& VIEL, J., 1999. Disease mapping and its uses. In: Disease Mapping and Risk Assessment for Public Health (A. Lawson, A. Biggeri, D. Böhning, E. Lesaffre, J. Viel \& R. Bertollini, ed.), pp. 3-13, New York: John Wiley \& Sons.

LEAL, M. C. \& SZWARCWALD, C. L., 1997. Características da mortalidade neonatal no Estado do Rio de Janeiro na década de 80: Uma visão espaçotemporal. Revista de Saúde Pública, 31:457-465.

MARSHALL, R. J., 1991. Mapping disease and mortality rates using empirical Bayes estimators. $A p$ plied Statistics, 40:283-294. 
MELLO-JORGE, M. H. P.; GOTLIEB, S. L. D. \& OLIVEIRA, H., 1996. O sistema de informação sobre nascidos vivos: Primeira avaliação dos dados brasileiros. Informe Epidemiológico do SUS, 5:15-48.

MELLO-JORGE, M. H. P.; GOTLIEB, S. L. D.; SOBOLL, M. L. M. S; ALMEIDA, M. F. \& LATORRE, M. R. D. O., 1993. Avaliação do sistema de informação sobre nascidos vivos e o uso de seus dados em epidemiologia e estatísticas de saúde. Revista de Saúde Pública, 27(Sup.):1-44.

MONTEIRO, C. A., 1982. Contribuição para o estudo do significado da evolução do coeficiente de mortalidade infantil no município de São Paulo-SP (Brasil) nas três últimas décadas: (1950-1979). Revista de Saúde Pública, 16:7-18.

MONTEIRO, C. A.; BENÍCIO, M. H. D. A. \& BALDIJÃO, M. F. A., 1980. Mortalidade no primeiro ano de vida e a distribuição de renda e de recursos públicos de saúde. São Paulo (Brasil). Revista de Saúde Pública, 14:515-539.

MORAIS NETO, O. L. \& BARROS, M. B. A., 2000. Fatores de risco associados à mortalidade neonatal e pós-neonatal na Região Centro-Oeste do Brasil: Linkage entre bancos de dados de nascidos vivos e óbitos infantis. Cadernos de Saúde Pública, 16: 477-485.

PAIM, J. S. \& COSTA, M. C. N., 1987. Spatial distribution of proportional infant mortality and certain socioeconomic variables in Salvador, Bahia, Brazil. PAHO Bulletin, 21:225-235.

PAIM, J. S. \& COSTA, M. C. N., 1993. Decréscimo e desigualdade da mortalidade infantil: Salvador, 1980-1988. Boletín de la Oficina Sanitaria Panamericana, 114:415-428.
PAIM, J. S., 1997. Abordagens teórico-conceituais em estudos de condições de vida e saúde: Notas para reflexão e ação. In: Condições de Vida e Situação de Saúde (R. B. Barata, org.), pp. 7-30, Rio de Janeiro: ABRASCO.

SANTOS, M., 1996. Por uma Geografia Nova. 4a Ed. São Paulo: Editora Hucitec.

SOUZA, A. C. T.; CUFINO, E.; PETERSON, K. E.; GARDNER, J.; AMARAL, M. I. V. \& ASCHERIO, A., 1999. Variations in infant mortality rates among municipalities in the state of Ceará, Northeast Brazil: An ecological analysis. International Journal of Epidemiology, 28:267-275.

TANGO, T., 1999. Comparisons of general tests for spatial clustering. In: Disease Mapping and Risk Assessment for Public Health (A. Lawson, A. Biggeri, D. Böhning, E. Lesaffre, J. Viel \& R. Bertollini, ed.), pp. 111-117, New York: John Wiley \& Sons.

XIMENES, R. A. A.; MARTELLI, C. M. T.; SOUZA, W. V.; LAPA, T. M.; ALBUQUERQUE, M. F. M.; ANDRADE, A. L. S. S.; MORAIS NETO, O. L.; SILVA, S. A.; LIMA, M. L. C. \& PORTUGAL, J. L., 1999. Vigilância de doenças endêmicas em áreas urbanas: A interface entre Figuras de setores censitários e indicadores de morbidade. Cadernos de Saúde Pública, 15:53-61.

ZOELLNER, I. K. \& SCHMIDTMANN, I. M., 1999. Empirical studies of cluster detection - Different cluster tests in apliccation to german cancer maps. In: Disease Mapping and Risk Assessment for Public Health (A. Lawson, A. Biggeri, D. Böhning, E. Lesaffre, J. Viel \& R. Bertollini, ed.), pp. 169-178, New York: John Wiley \& Sons. 